Classification

Physics Abstracts

$61.60-61.80 \mathrm{~F}-61.16 \mathrm{D}$

\title{
Oxygen segregation on a dislocation core in germanium studied by electron energy loss spectroscopy
}

\author{
A. Bourret \\ Centre d'Etudes Nucléaires de Grenoble, Département de Recherche Fondamentale, \\ Section de Physique du Solide, 85X, 38041 Grenoble Cedex, France \\ C. Colliex and P. Trebbia \\ Laboratoire de Physique du Solide, Université de Paris Sud, 91405 Orsay, France
}

(Reçu le 23 septembre 1982, accepté le 17 novembre 1982)

\begin{abstract}
Résumé. - Des analyses à l'échelle du nm sur le cœur de dislocation dans le germanium ont mis en évidence la présence de l'oxygène : quelques centaines d'atomes d'oxygène à une concentration moyenne de $5 \%$ sont détectés grâce à un microscope électronique à balayage muni d'un filtre de vitesse. Une désorption d'oxygène induite par les électrons est observée sous le faisceau. Ce phénomène est interprété comme étant la succession d'un processus d'ionisation puis de diffusion assistée par choc à l'interface oxyde-germanium.
\end{abstract}

\begin{abstract}
Nanoanalyses performed on a dislocation core in germanium give evidence of oxygen precipitation : a few hundreds of oxygen atoms at a mean concentration of $5 \%$ were detected. A radiation induced oxygen desorption is also observed under the electron beam for which a mixed knock-on and ionization process is proposed. This phenomenon is the main limiting factor for a more quantitative analysis.
\end{abstract}

1. Introduction. - Impurity segregation on dislocations in Czochralsky (CZ) grown germanium or silicon crystals has been recently reported [1] on the basis of high resolution electron microscopy (HREM) observations. This phenomenon is of great importance in semiconductor materials and is commonly used as a gettering process to trap impurities. It is not possible to determine the chemical nature of such impurities by HREM. The precipitates to be analysed are spatially highly concentrated within a few $\AA$ around the dislocation cores and the total number of impurity atoms is small. A nanoanalysis concerning such small volumes can only be achieved with a dedicated field emission scanning transmission electron microscope (STEM). The X-ray spectra recorded from these precipitates do not show any characteristic lines due to impurity atoms with $Z>9$ [2]. It becomes necessary to use electron energy loss spectroscopy (EELS) which is the only available method to detect low $Z$ elements.

2. Experimental results. - Various subgrain tilt boundaries with a common [011] axis were first examined by HREM. When viewed along [011], dislocations are seen end-on and the projected 
image enables us to directly determine the projected Burgers vector, the splitting distance between partial dislocations or the exact core structure [3,4]. The electron flux employed during these observations is equal to $J_{0}=4 \mathrm{~A} / \mathrm{cm}^{2}$ and no electron damage is ever observed. The results already reported are very similar in $\mathrm{CZ}$ germanium and silicon, but germanium specimens have been selected for the present work because they are much less sensitive to spontaneous surface oxidation. A large variety of dislocation arrangements is present in a low angle grain boundary. Some of them contain a very well defined second phase in their core with an abrupt interface. This phase appears crystalline and is continuous all along the dislocation. The precipitate size as directly measured from HREM is essentially a function of the Burgers vector (Table I).

Table I. - Precipitate sizes, $d$, and estimated number of impurity atoms, $N_{0}$, (per $100 \AA$ of dislocation length) as a function of the Burgers vector, $\mathbf{b}$.

\begin{tabular}{ccccc}
\hline b & $\begin{array}{c}1 / 6[2 \overline{1} 1] \\
\text { Schockley }\end{array}$ & $\begin{array}{c}1 / 2[0 \overline{1} 1] \\
\text { Lomer }\end{array}$ & $\begin{array}{c}1 / 3[11 \overline{1}] \\
\text { Frank }\end{array}$ & {$[100]$} \\
\hline$d(\AA)$ & invisible & $5-8$ & $7-9$ & $15-25$ \\
$N_{0}$ & $\leqslant 30$ & $55-140$ & $100-170$ & $500-1350$ \\
\hline
\end{tabular}

The number of impurity atoms $N_{0}$ is evaluated assuming a precipitate formula $\mathrm{GeX}_{2}$ and a structure isomorphic to coesite [1]. It is clear from table I that it is important to perform the ana-
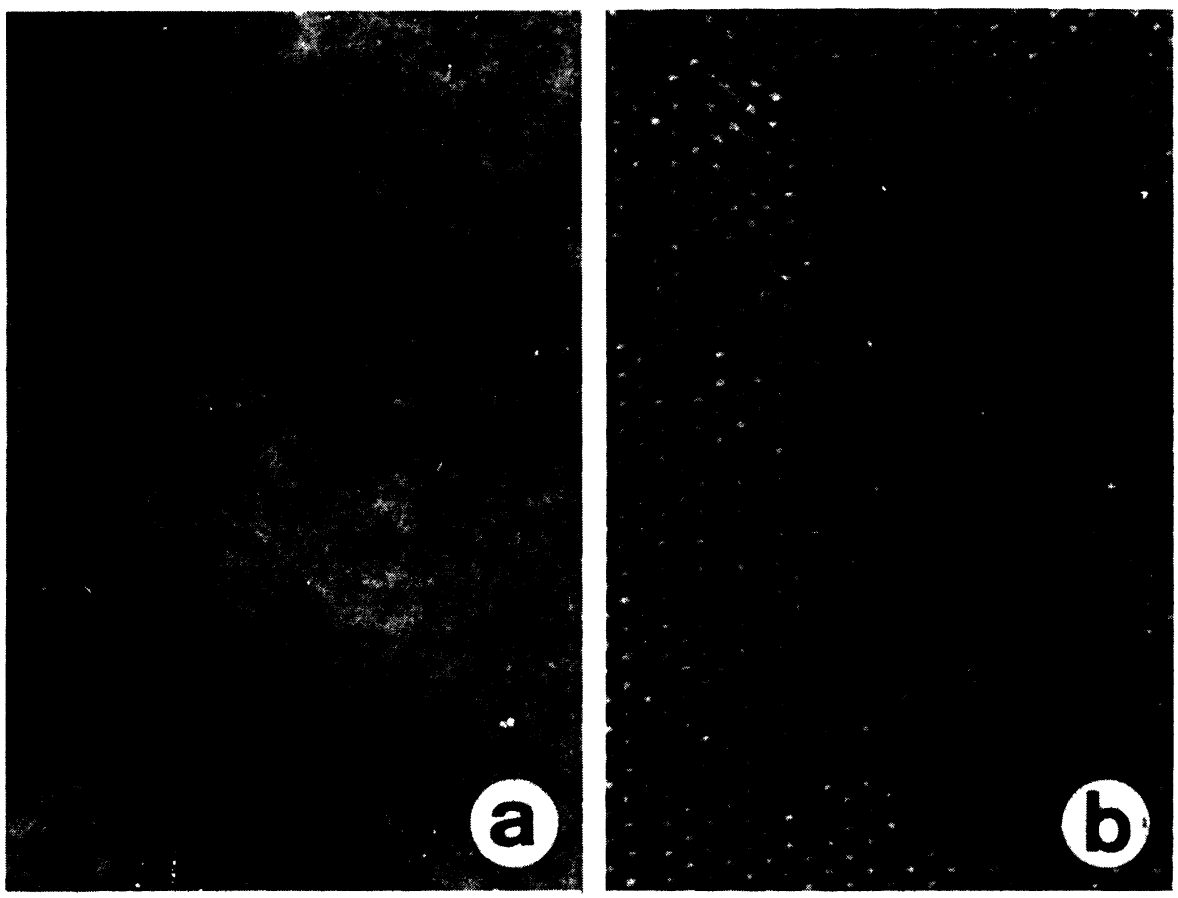

Fig. 1. - Dislocations A and B analysed by EELS. $a$ ) STEM bright field image before the analysis; $b$ ) same dislocations after the analysis as viewed by HREM. Dislocation A has received $5.8 \times 10^{4} \mathrm{C} / \mathrm{cm}^{2}$ and dislocation $\mathrm{B}=1.2 \times 10^{4} \mathrm{C} / \mathrm{cm}^{2}$. The dislocation $\mathrm{A}$ has been cleaned up after electron induced oxygen desorption. 
lysis on the largest precipitates occurring on the [100] type dislocation. The selection of these specific dislocations is achieved directly in the STEM : a characteristic moire pattern is visible on the precipitate (Fig. 1a). Once the nanoanalysis has been performed the same sample is transferred back to a $200 \mathrm{keV}$ transmission electron microscope in the HREM mode. Structural images are then taken on the same dislocation previously analysed (Fig. $1 b$ ). They reveal that precipitates are affected by the electron beam in the STEM. The precipitate on dislocation A (initial impurity atom number $N_{0_{1}} \sim 1000$ ) completely disappears after an electron dose of $5.8 \times 10^{4} \mathrm{C} / \mathrm{cm}^{2}$. Similarly the precipitate on dislocation $\mathrm{B}\left(N_{0_{2}} \sim 500\right)$ is reduced in size down to $N \sim 100$ atoms after an electron dose of $1.2 \times 10^{4} \mathrm{C} / \mathrm{cm}^{2}$. The specimen thickness is measured by stereomicroscopy at the dislocation location and is determined to be $90 \pm 10 \AA$. A small apparent thinning of the specimen is noted on all analysed dislocations.

The EELS nanoanalysis has been achieved with a VG-HB5 microscope fitted with a Gatan spectrometer collecting a large angular distribution of inelastic electrons [5] (half angle $\sim 20 \mathrm{mrad}$ ). The incident $100 \mathrm{keV}$ electrons are focused in a $5 \AA$ diameter probe carrying a current of $6 \times 10^{-11} \mathrm{~A}$. This probe is permanently scanned over the selected reduced area of $45 \AA \times 30 \AA$ size giving an average flux $J_{0}=4.4 \times 10^{2} \mathrm{~A} / \mathrm{cm}^{2}$. After a quick scan over the whole 0 to $1 \mathrm{kV}$ spectrum, repetitive scans are performed on a restricted energy domain around the ionization edges of interest (nitrogen K edge : $405 \mathrm{eV}$, oxygen $\mathrm{K}$ edge : $532 \mathrm{eV}$ ). Due to the weak signal and the short life time of the precipitate the recorded spectra are very noisy. Two typical examples comparing a selected area on the matrix and on one of the precipitates are shown in figure 2 . To
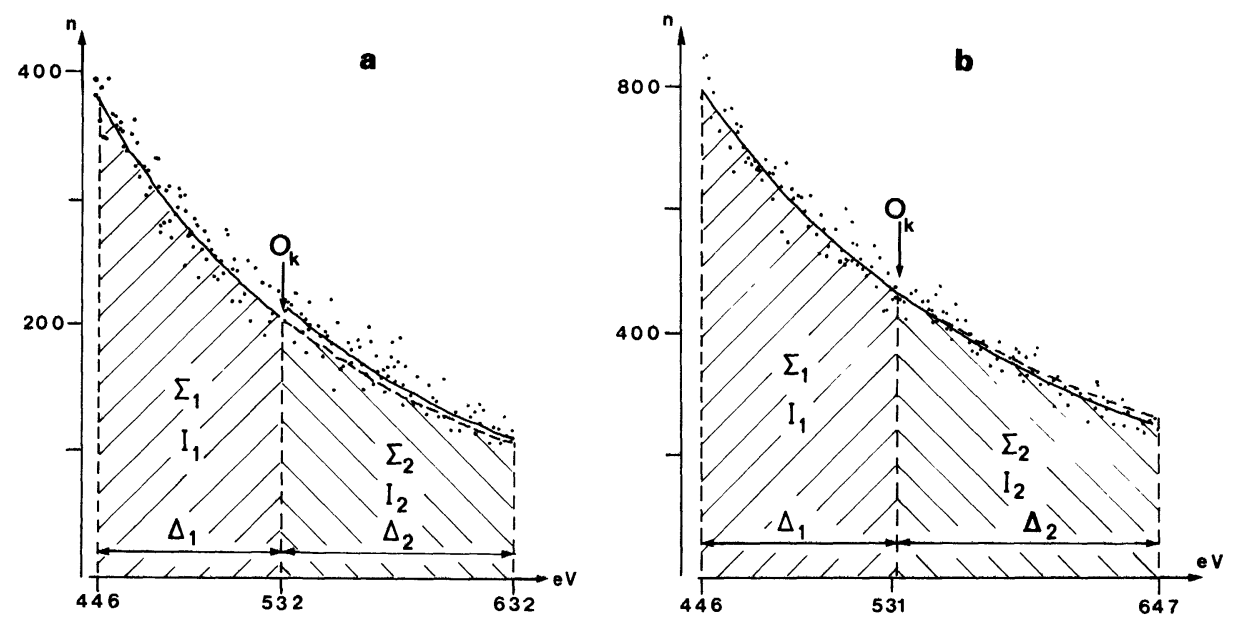

Fig. 2. - EELS spectra recorded on the dislocation A (a) and on the matrix (b). The fit with $A \Delta E^{-r}$ curve before the oxygen $\mathrm{K}$ edge is extrapolated after the edge defining $I_{2}$, which is compared to the total number of experimental counts $\Sigma_{2}$ on the same energy window $\Delta_{2}$.

extract a more quantitative information about the oxygen content the following data analysis is made. A model background of type A $\Delta E^{-r}$ is fitted with the parental distribution of data points over an energy window $\Delta_{1}$ before the edge following " curfit " program [6]. One compares the actual number of counts $\Sigma_{1}$ with the area $I_{1}$ under the fit. The very good agreement between the $I_{1}$ and $\Sigma_{1}$ values constitutes a test of the accuracy of the fitting procedure. The background model is then extrapolated over an energy window $\Delta_{2}$ below the edge of interest and the characteristic signal is measured as $S=\Sigma_{2}-I_{2}$ where $\Sigma_{2}$ is the total number of experimental counts and $I_{2}$ 
the fit area. This signal has to be compared with the statistical uncertainty $\sigma_{\text {s }}$, roughly expressed by [7] :

$$
\sigma_{\mathrm{s}}=\sqrt{\chi_{\min }^{2}\left[I_{2}+\Sigma_{2}\right]}
$$

where $\chi_{\min }^{2}$ is the standard least square deviation. Table II shows typical set of results for the analysis of oxygen and nitrogen. More than 6 runs all show similar results on different dislocations and samples.

Table II. - Total count number before and after the edge, $\Sigma_{1}$ and $\Sigma_{2}$ compared to the best background fit $I_{1}$, and the extrapolated fit $I_{2}$. This gives the signal, $S$, for dislocation A (oxygen) and dislocation $\mathrm{B}$ (oxygen and nitrogen).

\begin{tabular}{|c|c|c|c|c|c|c|c|}
\hline \multirow[t]{2}{*}{ Probe localized on } & \multicolumn{2}{|c|}{ Before the edge } & \multicolumn{2}{|c|}{ After the edge } & \multirow{2}{*}{$\begin{array}{c}\text { Signal } \\
S=\Sigma_{2}-I_{2}\end{array}$} & \multirow{2}{*}{$\begin{array}{c}\text { Statistical } \\
\text { uncertainty } \\
\sigma_{\mathrm{s}}\end{array}$} & \multirow{2}{*}{$\begin{array}{c}\text { Dose for } \\
\text { analysis } \\
D\left(\mathrm{C} / \mathrm{cm}^{2}\right)\end{array}$} \\
\hline & $\Sigma_{1}$ & $I_{2}$ & $\Sigma_{2}$ & $I_{2}$ & & & \\
\hline Matrix & 51697 & 51687 & 39922 & 40696 & -774 & 284 & $2 \times 10^{4}$ \\
\hline Dislocation $\mathrm{A}\left(\mathrm{O}_{\mathbf{k}}\right)$ & 155852 & 155789 & 113459 & 111549 & 1910 & 543 & $5.8 \times 10^{4}$ \\
\hline Dislocation $\mathrm{B}\left(\mathrm{O}_{\mathrm{k}}\right)$ & 25251 & 25241 & 19437 & 18670 & 767 & 195 & $1.2 \times 10^{4}$ \\
\hline Dislocation $\mathbf{B}\left(\mathrm{N}_{\mathrm{k}}\right)$ & 30654 & 30653 & 36643 & 36479 & 164 & 270 & $1.2 \times 10^{4}$ \\
\hline
\end{tabular}

It is deduced that there is no detectable presence of oxygen on all the runs registered on the matrix. The negative value of the signal confirms that the simple power law model is not quite valid when extrapolated over $200 \mathrm{eV}$ energy range [8], the $r$ parameter increases slightly and regularly with the energy loss. The chemical analysis on the dislocation cores has to be discussed in terms of a probability function that the signal is due to elemental detection or to normal error distribution. By reference to a table of integrals of Gaussian distribution for the value $S / \sigma_{\mathrm{s}}$, one estimates the probability of having oxygen in dislocation cores A and B greater than $99.9 \%$ and the probability of nitrogen on dislocation B of the order of $46 \%$. It confirms the previous assumption that the precipitates are oxides. A further confirmation of the reliability of our statistical procedure is obtained by applying to run A or B similar analysis searching for the presence of $\mathrm{V}$ or $\mathrm{Cr}$. Although their ionization edges $(513 \mathrm{eV}$ and $574 \mathrm{eV})$ are very close to the oxygen $\mathrm{K}$ edge, the corresponding signal was always negative and inferior to the statistical uncertainty. An order of magnitude of the number of oxygen atoms, $N$, involved in the present analysis can be estimated from the formula :

$$
S=J_{0} \sigma_{\mathrm{K}} N \eta \tau \delta_{\text {slit }} / \delta_{\text {step }}
$$

where $\tau$ is the counting time per channel, $\delta_{\text {slit }}$ and $\delta_{\text {step }}$ the respective energy width of the slit detector and the energy increment between channels.

For the very thin germanium foils and large angles of collection concerned in this analysis, $J_{0}$ is roughly equal to the primary electron flux [9]. The cross section, $\sigma_{\mathrm{K}}$, is estimated of the order of $2 \times 10^{-21} \mathrm{~cm}^{2}$ by Egerton [10] for a $100 \mathrm{eV}$ energy window. The detection efficiency, $\eta$, is $\simeq 1$ and $\delta_{\text {slit }} / \delta_{\text {step }} \simeq 4$. The insertion of the experimental values shown in the table II for dislocation B leads to an estimate of $N$ of the order of several hundreds, corresponding to an average concentration of $5 \%$ in the analysed volume. 
3. Discussion. - The most important limiting factor is the gradual disappearance of the $\mathrm{GeO}_{2}$. The oxygen mobility is not due to a thermally induced process. A rough estimate of the maximum temperature increase, $\Delta T$ under the beam is given by the Reimer formula [11] :

$$
\Delta T \sim I T / 2 K_{\mathrm{T}} \log r_{\mathrm{g}} / r_{\mathrm{b}}
$$

with $I=$ total current, $\mathfrak{T}=$ stopping power at the incident electron energy, $K_{\mathrm{T}}=$ thermal conductivity, $r_{\mathrm{g}}=$ outside radius of the specimen at the reference temperature and $r_{\mathrm{b}}=$ beam radius. Application of (3) gives a negligeable temperature rise in the STEM conditions $\left(\sim 6 \times 10^{-3}{ }^{\circ} \mathrm{C}\right)$ and a small heating effect in the HREM conditions $\left(5^{\circ}\right)$ where no radiation effect is observed however. The oxygen atoms must have escaped from the foil : similar phenomenon has already been observed on electron irradiated $\mathrm{SiO}_{2}$ surfaces by Auger electron spectroscopy [12]. Quantitatively we can assume an exponential decay of the number of oxygen atoms $N$, present in the precipitate with initial number $N_{0}$, given by :

$$
N=N_{0} \exp -D / D_{0}=N_{0} \exp -\sigma_{0} D
$$

where $D$ is the dose received after a time $t$ and $D_{0}$ a characteristic dose equal to the inverse of the total apparent cross section for removal of an oxygen atom $\sigma_{0}$.

Using the experimental data on dislocation A and B leads to $D_{0} \simeq 0.75 \times 10^{4} \mathrm{C} / \mathrm{cm}^{2}$ and $\sigma_{0}=21$ barns. This low cross section has to be compared to the much higher value found for removing oxygen atoms from the surface of silicon [13] which is of the order of 400 barns if scaled for $100 \mathrm{kV}$ electrons. This indicates that the radiation damage speed is controlled by a much slower mechanism than ionization. Therefore a model including knock-on processes and ionization is proposed. It includes the following steps : i) production of oxygen interstitials close to the Ge$\mathrm{GeO}_{2}$ interface either by direct knock-on or Coulomb explosion following ionization; ii) radiation induced diffusion of oxygen in the interface towards the free surface; iii) radiation induced desorption of oxygen after ionization close to the surface. The maximum energy transfer by $100 \mathrm{kV}$ electrons to oxygen atoms is $\sim 15 \mathrm{eV}$, which can explain correctly the first two steps [14] with a relatively low cross section. It must be remarked that none of the steps are thermally activated and may occur at room temperature.

\section{References}

[1] Bourret, A., Desseaux, J. and D'Anterroches, C., in Inst. Phys. Conf. Ser. no ${ }^{\circ}$, The Institute of Physics (1981) 9.

[2] Bourret, A. and Colliex, C., submitted for publication in Ultramicroscopy.

[3] Bourret, A., Desseaux, J. and Renault, A., Philos. Mag. A-45 (1982) 1.

[4] Bourret, A., Desseaux, J., Philos. Mag. A-39 (1979) 419.

[5] Jeanguillaume, C., Krivanex, O. and Colliex, C., in Inst. Phys. Conf. Ser. ${ }^{\circ}$ 61, The Institute of Physics (1981) 189.

[6] Colliex, C., Jeanguillaume, C. and Trebbia, P., in Microprobe Analysis of biological systems (Academic Press) 1981, p. 251.

[7] Bevington, P. R., Data reduction and error analysis for the physical sciences (Mc Graw Hill) 1969.

[8] Trebbia, P. and Colliex, C., Inst. Phys. Conf. Ser. no 52 (1980) 333.

[9] EgERTon, R. F., Ultramicroscopy 3 (1978) 243. 
[10] Egerton, R. F., J. Micros. 123 (1981) 333.

[11] REIMER, L., Elektronenmikroskopische Untersuchungs- und Präparations methoden (Springer) 1967, p. 226.

[12] Carrière, B. and Lang, B., Surf. Sci. 64 (1977) 209.

[13] Thomas, S., J. Appl. Phys. 45 (1974) 161.

[14] Cosslett, V. E., Inst. Phys. Conf. Ser. no 52 (1980) 277. 